\title{
OBJECT-BASED IMAGE ANALYSIS OF DIFFERENT SPATIAL RESOLUTION SATELLITE IMAGERIES IN URBAN AND SUBURBAN ENVIRONMENT
}

\author{
I. Kotaridis ${ }^{1} *$, M. Lazaridou ${ }^{1}$ \\ ${ }^{1}$ Aristotle University of Thessaloniki, Faculty of Engineering, School of Civil Engineering, \\ Lab. of Photogrammetry - Remote Sensing, 54124 Thessaloniki, Greece - (iskotarid, lazamari)@ civil.auth.gr
}

Commission III, WG III/1

KEY WORDS: OBIA, Image segmentation, SVM, Landsat-8, Sentinel-2, QuickBird, Land cover classification

\begin{abstract}
:
Monitoring urban and suburban land cover has become a particularly researched investigation field in remote sensing community, since there is a large amount of professionals interested in gathering useful information, regarding urban sprawl and its side effects in natural vegetation, urban parks and water bodies. This paper focuses on studying the implementation of an object-based image analysis methodological framework, in Orfeo ToolBox. Moderate, high and very high spatial resolution satellite images were utilized in order to generate thematic land cover maps of the study area located in Thessaloniki, Greece. Taking into consideration that there is not a relevant research in literature concerning the selection of segmentation parameters values, the optimal values are presented for MeanShift segmentation algorithm. Classifications were conducted with the use of Support Vector Machines algorithm and the final outputs are presented, accompanied by the evaluation of accuracy assessments which is a mandatory step in every remote sensing project. The analysis showed that OBIA, in this case, works well with Landsat- 8 and QuickBird data and exceptionally well with Sentinel-2A data with over $90 \%$ overall accuracy. Critical considerations on the aforementioned are also included.
\end{abstract}

\section{INTRODUCTION}

It is widely accepted that valid geospatial information are in high demand and need to be updated regularly. In this context, various satellite imagery manipulation approaches have been deployed including Object-Based Image Analysis (OBIA). It has the main goal of providing sufficient and automated procedures in order to analyze the imagery by using spectral, textural, spatial and topological characteristics (Blaschke et al., 2008).

OBIA has been developed since the establishment of very high spatial resolution imagery, where the pixel size is notably smaller than the object concerned. Through image segmentation, the preliminary stage of the procedure, the level of unnecessary detail decreases, the image complexity is reduced and the image content becomes more understandable. The products of this procedure are the image objects which are the main methodological component of object-based image analysis and represent a real-world object, whilst a pixel cannot (Blaschke et al., 2008). One of the main goals of merging pixels into objects is to get rid of the "salt and pepper" noise (Blaschke, 2010).

Object-based techniques assume that a single pixel is very likely to be associated with its neighbouring pixels and as consequence to belong to the same cluster. A first step is to partition the image into individual objects that share the same characteristics. In general, OBIA follows a procedure close to visual interpretation, taking into account the texture, shape and size of the objects, that represent real world entities. Although computer vision advances rapidly, it is still far from the entire potential of human perception. Segmentation is the very crucial first step in OBIA, since all following steps and results acquired are depended on segmentation performance (Nussbaum and Menz, 2008).

OBIA was applied in a number of application fields that emerged through the years, including forests, wetlands, urban areas, public health infrastructure and land use/land cover. Understanding the pattern that a landscape changes over time continues to be an important research field in remote sensing. The application of OBIA in order to study and understand this pattern is increasingly popular. But, despite the technological and methodological progress including object-based techniques, it has to be stated that pixel-based methods are represented far more in publications at present (Thenkabail, 2016).

The image segmentation algorithm provides a more clear understanding of the landscape and is a key feature so as to increase the accuracy of an image classification. The selection of the segmentation algorithm is mainly relevant to the target of the image analysis application. The outcome of segmentation approach is a product consisting of image objects that can be distinguished with labels that have no meaning at this time (e.g. object1, object). These labels have to be converted into substantial ones by an object-based classification (Thenkabail, 2016). Moreover, segments have supplementary spectral information in comparison with single pixels including mean values and median values per band as well as variance values (Blaschke, 2010).

Urban and suburban landscapes consist of various materials such as concrete, asphalt, metal, glass, water, grass, trees and soil organized by people in complicated patterns in order to build houses, commercial properties and land-based transport

\footnotetext{
* Corresponding author
} 
systems (Jensen and Cowen, 1999). Suburban areas, taking into account their close connection with the city, are highly related to the urban sprawl and its negative effects, such as the loss of agricultural land, natural vegetation, water bodies and the deterioration in the air quality due to the escalation in traffic congestion (Pozzi and Small, 2001). A large number of public services, organizations and professionals needs current data regarding urban and suburban infrastructure. They spend money on obtaining aerial imagery as well as other remotely sensed data to extract the needed land information, due to the fact that much of the aforementioned data cannot be acquired through in situ surveys (Jensen and Cowen, 1999).

Monitoring land cover change with a focus on urban parks, natural vegetation, water bodies has become a notably researched field of investigation caused by the huge consequence that land cover can have on a city's everyday life. For that purpose optical satellite imageries are utilized broadly. A large number of pixel-based and object-based methods have been investigated, using very high spatial resolution images (e.g. QuickBird) and moderate resolution images (e.g. Landsat). Since the launch of Sentinel-2,10m spatial resolution images have become freely available, providing an additional dataset in order to map land cover changes (Labib and Harris, 2018). OBIA has gained an increased popularity over the last few years due to the noteworthy classification accuracy results when applied to very high spatial resolution images (Csillik and Belgiu, 2017). However, OBIA has also been applied in land cover classifications of Landsat OLI imagery by employing new machine learning methods (e.g. Random Forests, SVM) with very good overall accuracy values when mapping urban, agricultural, forest areas (Phiri and Morgenroth, 2017) and large-scale seagrass mapping (Topouzelis et al., 2018). In addition, Sentinel-2 images were used for the application of OBIA on cropland mapping and monitoring (Csillik and Belgiu, 2017) and water body extraction (Kaplan and Avdan, 2017), greenhouse detection with remarkable accuracy values. Both
Landsat-8 OLI and Sentinel-2 MSI data were utilized in order to detect greenhouses (Novelli et al., 2016).

This study is focused on investigating the performance of an object-based image analysis workflow using a moderate, high and very high spatial resolution scene, so as to produce a thematic land cover map, while highlighting the segmentation parameters selected for each image. OBIA was considered as an appropriate choice, taking into account the limitations of a pixel-based image analysis (e.g. salt and pepper noise, priority to spectral values).

Support vector machine (SVM) is a non-parametric supervised spectral classification algorithm that is a popular choice when dealing with multispectral images. SVM aims to distinguish the training samples that belong to separate classes by identifying the maximum-margin hyperplanes in the space that the samples are mapped. The effective use of SVM in pixel-based approaches has inevitably led to its use in object-based methods. This approach turned to be more effective rather than other non-parametric classifiers (e.g. neural networks) regarding classification accuracy, computational complexity and robustness to parameter setting (Thenkabail, 2016). Nonparametric classifiers (e.g. SVM, ANN) produce better results than parametric classifiers when dealing with complex landscapes (Phiri and Morgenroth, 2017).

\section{STUDY AREA AND SATELLITE DATA}

\subsection{Study area}

The study area that was selected for this research is located in the north part of mainland Greece. A subset was extracted from the main scenes for analysis that includes a part of the regional unit of Thessaloniki, as it appears in Figure 1, which is presented below.

The selection of the study area was based on the diversification of land cover in the landscape that it presents. The urban area of

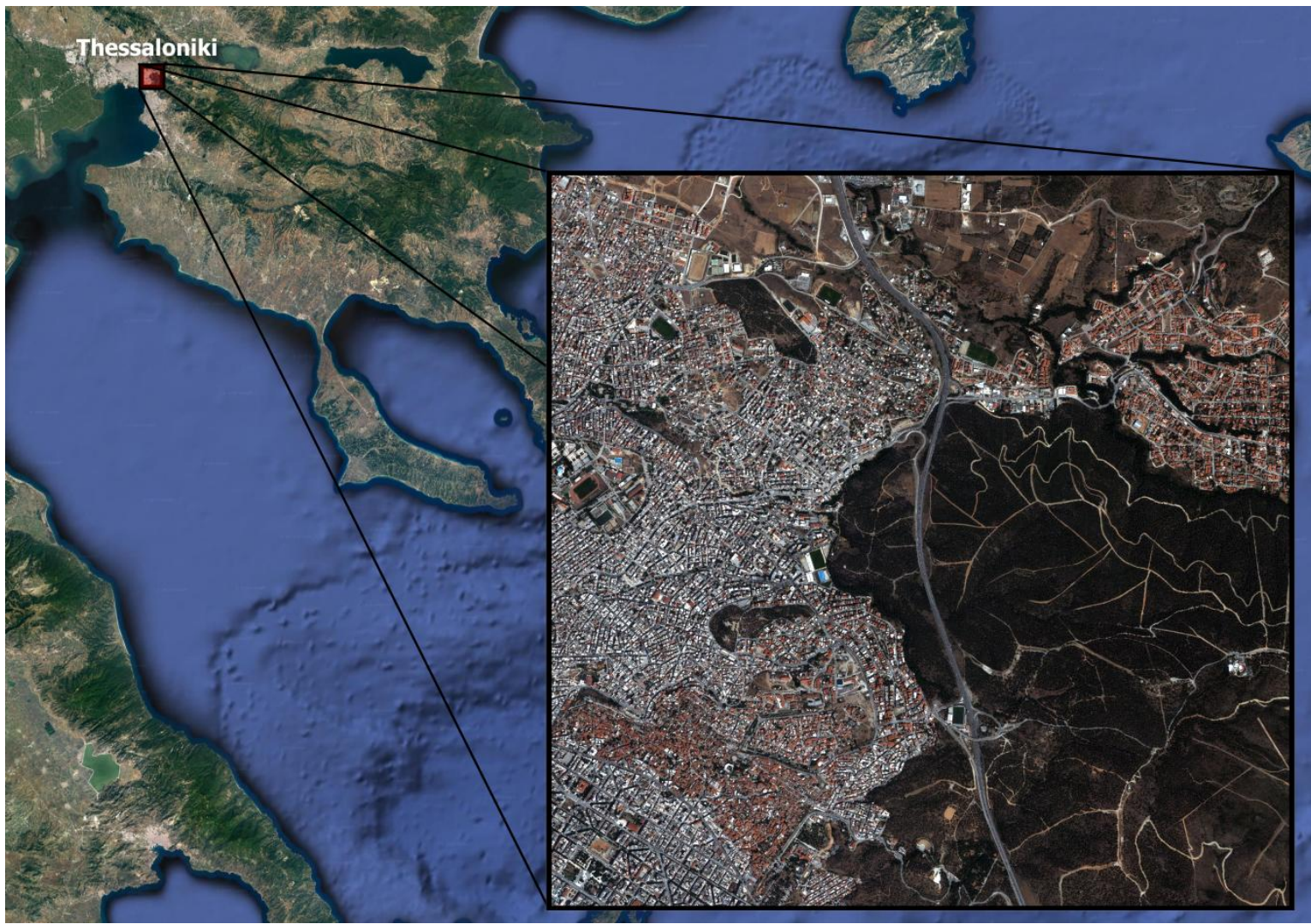

Figure 1. Study area in Google Maps (left) and Quickbird Pansharpened image of the year 2010 (right) (Source: Google

Maps, modified by the author)
This contribution has been peer-reviewed.

https://doi.org/10.5194/isprs-archives-XLIII-B3-2020-105-2020 | @ Authors 2020. CC BY 4.0 License. 
Thessaloniki is located on the left side of the subset image, while the suburban area is located on the top right corner of the image. A man made border between those two different landscapes, the ring road of Thessaloniki, can also be identified. Moreover, agricultural land can be detected on the top of the scene, while a large part of the study area is occupied by forest. These heterogeneous types of land cover compose an interesting urban and suburban landscape that transforms through the years.

\subsection{Satellite data}

In this paper, three images were obtained with different spatial resolution each:

1. A Landsat-8 Surface Reflectance Level-2 image acquired 25 August, 2018 (Path 184, Row 32), with 30m spatial resolution.

2. A Sentinel-2A Level-2A Bottom-Of-Atmosphere (BOA) corrected reflectance image acquired 23 July, 2018 (Tile T34TFL).

3. A Quickbird Pansharpened Level 2A (Standard) image acquired 26 July, 2009, with $0,60 \mathrm{~m}$ spatial resolution.

The criteria for the selection of scenes were limited cloud coverage, high quality and close acquisition date of the same season. All Satellite images were atmospherically and geometrically corrected.

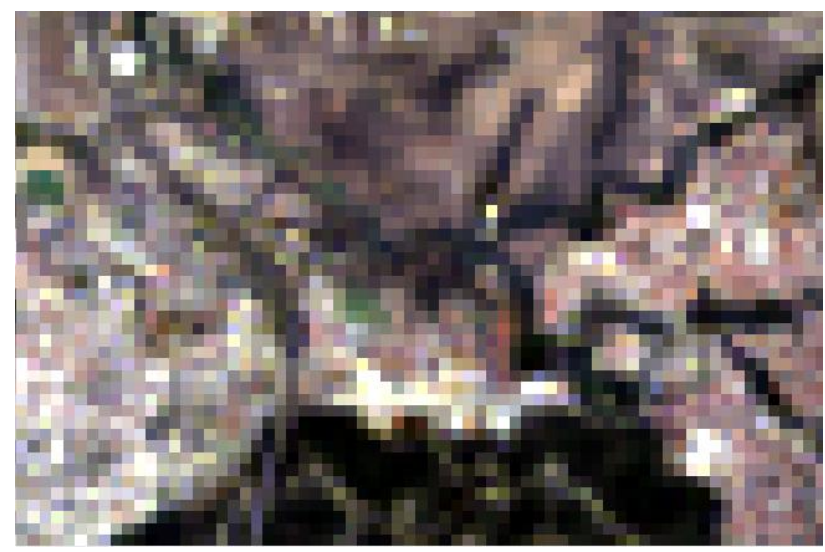

Figure 2. Subset of Landsat-8 image. Pixel size:30m

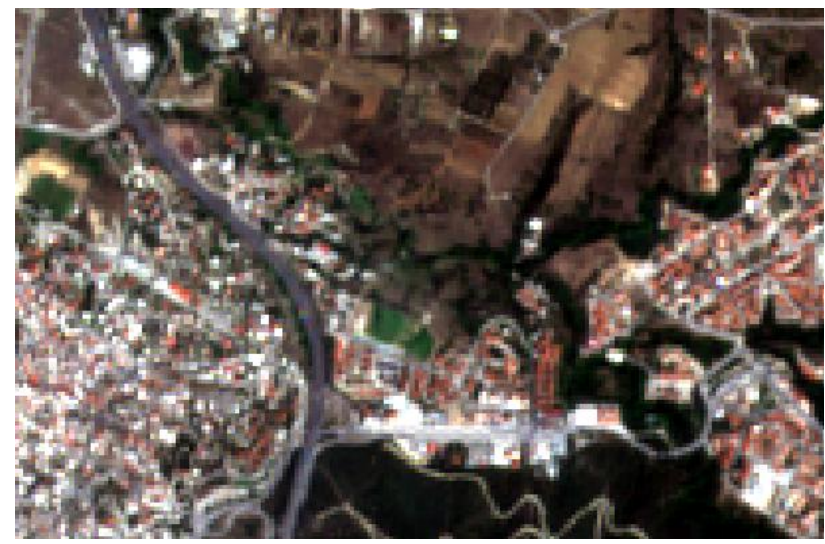

Figure 3. Subset of Sentinel-2A image. Pixel size:10m

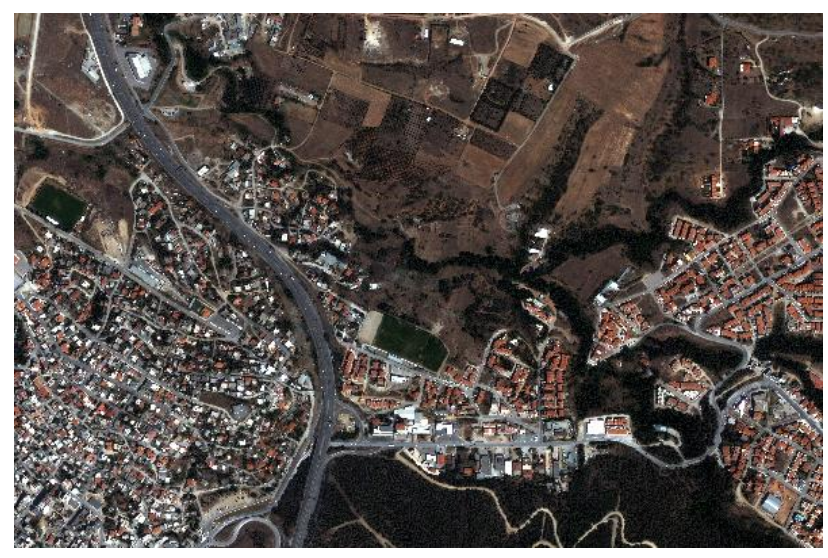

Figure 4. Subset of Quickbird image. Pixel size:0,6m

\section{METHODOLOGICAL FRAMEWORK - DATA PROCESSING}

\subsection{Tools}

Digital processing of the images was carried out in Orfeo ToolBox (OTB), a remote sensing oriented image analysis open-source project. OTB offers more than 90 applications regarding remote sensing tasks such as image access, geometric modeling, filtering, feature extraction, image segmentation, object extraction, classification and change detection (Christophe et al., 2008). Specifically, for the purpose of this study, the applications were accessed from Monteverdi, a lightweight satellite image rendering and processing tool that offers access to all processing applications from OTB. It does not aim to replace a GIS software that is more appropriate for editing and displaying raster and vector data (Grizonnet et al., 2017). In addition, QGIS was used in order to visualise the results, generate land cover maps and extract quantitative information.

\subsection{Methodology}

The diagram that is presented in Figure 5 outlines the objectbased image analysis methodological framework that was implemented in order to produce the classification products of the three images in the study area.

ConcatenateImages application was utilized to perform images multispectral bands concatenation. As a result, a single multichannel image was generated. It has to be noted that the Quickbird imagery was available in a 4-band Pansharpened product. This option integrates the visual information of the multispectral bands with the spatial information of the panchromatic one into a final single image product (DigitalGlobe, 2003). Thus, the concatenation was applied only to the Landsat and Sentinel imagery. 


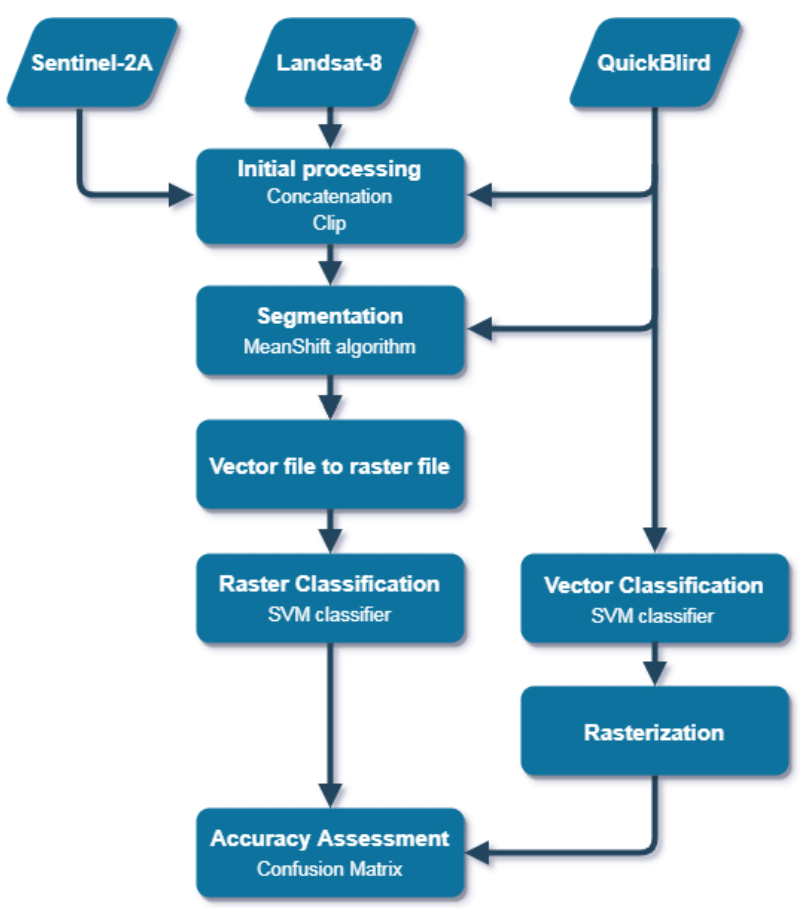

Figure 5. Object-Based Image Analysis methodological framework

Subsequently, in order to clip the scenes at the boundaries of the study area, a vector layer was created and the ExtractROI application was used. It extracts the region of interest with the selection among four modes. In particular, the fit mode was chosen which needs a reference image or vector file so as to crop the initial image to the same extend as the reference.

The outputs of the aforementioned process were used for segmentation process. Orfeo Toolbox offers user the option to perform a variety of segmentation algorithms including watershed, meanshift, connected components and morphological profiles. For the purpose of this study the MeanShift (MS) algorithm was selected. MS is a nonparametric clustering method which is applied extensively in image processing (Remote Sensing for Forest Cover Change Detection, 2016). MS has depicted superb results regarding object delineation (Chehata et al., 2014).

Orfeo ToolBox offers a separate application for this type of segmentation called Large-Scale MeanShift. It generates a vector output consisting of the regions extracted with the algorithm. A number of parameters can be selected during the process, among which spatial radius and range radius, that take into account the resolution of the image and the minimum segment size, that allows to get rid of small regions whose size is less than the given parameter, are of great importance. Spatial radius parameter defines the radius of the spatial neighbourhood for averaging. Setting high values to this parameter will lead to a smoother result but the processing time will be higher. Range radius parameter defines the threshold on spectral signature euclidean distance in order to examine neighbourhood pixel for averaging. Selecting higher values will result in less edgemaintaining, while lower values will lead to less noise smoothing. Changing this parameter has no consequence on processing time. Lastly, minimum segment size parameter set a boundary that if a segment has a size lower than this value, then it is grouped with the segment with the closest spectral signature (OTB CookBook, 2018). In the output file, which can have the form of a shapefile, there are fields that define each segment. Particularly, the radiometric mean and standard deviation for each band is computed.

It has to be underlined that there is not a suitable guide in literature regarding the parameters values selection. In order to determine the optimal parameters values, a long series of tests were performed. They included a trial and an error procedure on the basis of visual interpretation. The final parameters values of MeanShift segmentation algorithm are presented in Table 1.

\begin{tabular}{|c|c|c|c|}
\hline Image & $\begin{array}{c}\text { Spatial } \\
\text { radius }\end{array}$ & $\begin{array}{c}\text { Range } \\
\text { radius }\end{array}$ & $\begin{array}{c}\text { Minimum } \\
\text { Segment Size }\end{array}$ \\
\hline Landsat-8 & 5 & 15 & 15 \\
\hline Sentinel-2A & 5 & 19 & 75 \\
\hline QuickBird & 5 & 23 & 160 \\
\hline
\end{tabular}

Table 1. Optimal parameters values for MeanShift segmentation of the images

Saving segmentation outputs in vector files has several assets. An important one is that there are plenty of file formats that depict this type of vector data, particularly in GIS world. This provides full interoperability among the segmentation tools and almost all GIS software that is advantageous (Michel et al., 2012).

The vector files were converted to raster using zonal statistics. In this way, each raster output consists of the mean and the variance of the pixels within every segment in separate bands. In order to succeed this, a python script was run in OSGeo4W command line shell. The image file, the segment vector and the statistics to be calculated were entered in the script. This procedure produces a multiband image with the mean and variance for the pixels in a segment. A subset of the true colour composite (using mean value bands) for each image is presented below.

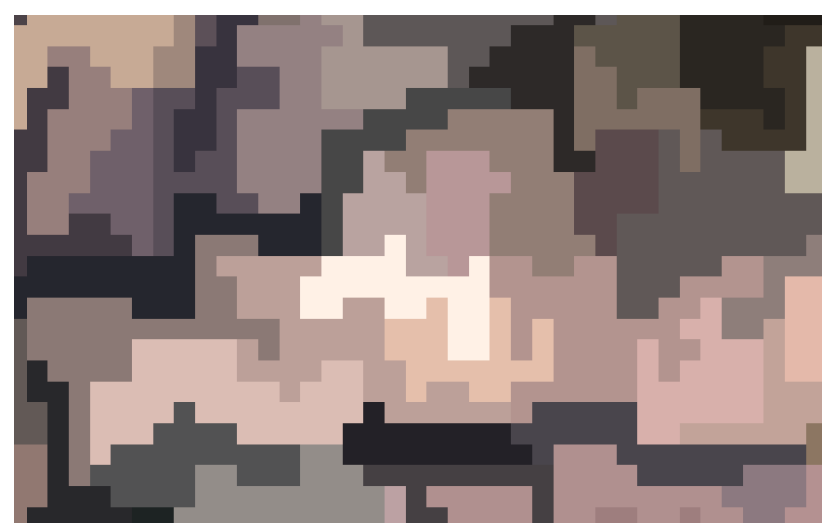

Figure 6. Subset of Landsat-8 segmented image 


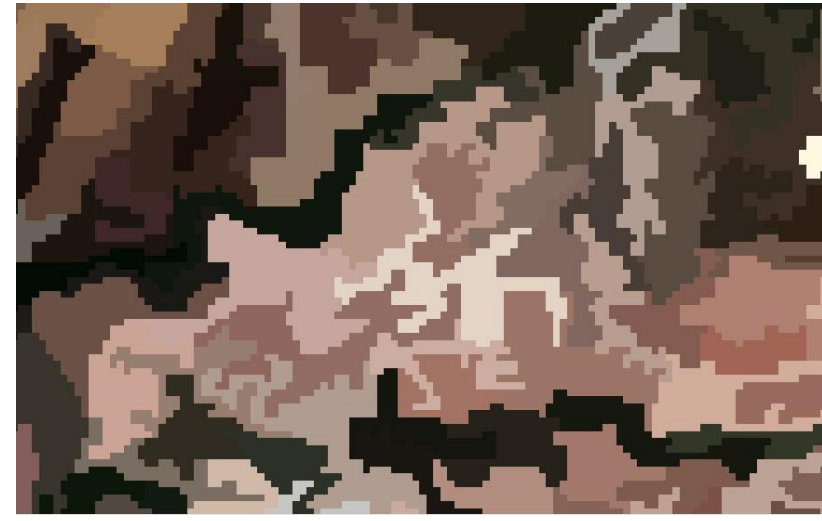

Figure 7. Subset of Sentinel-2A segmented image

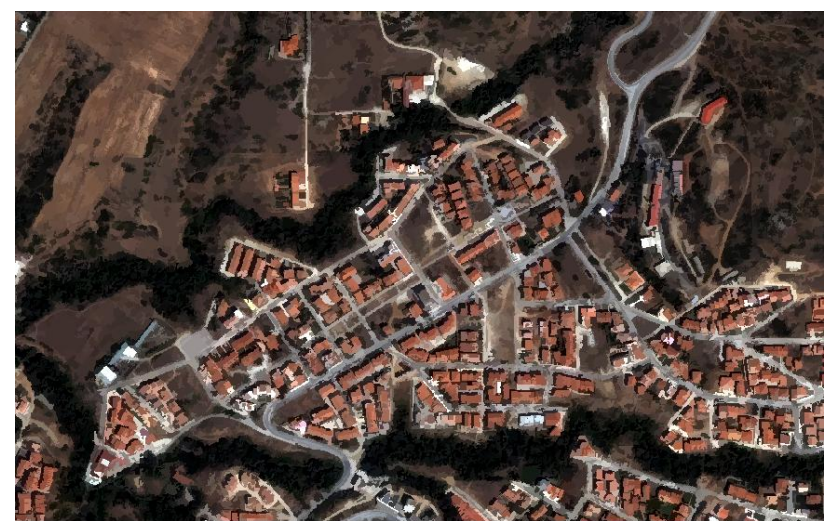

Figure 8. Subset of Quickbird segmented image

Landsat segmented image from a total of 17.955 pixels resulted in 650 segments, Sentinel image from a total of 160.792 pixels resulted in 1.138 segments and Quickbird image from a total of 44.726.535 pixels resulted in 141.191 segments. Taking this into consideration, a raster classification was performed for the Landsat- 8 and Sentinel-2A segmented images, while a vector classification was performed of the Quickbird image. It was not possible to perform a raster classification on the Quickbird image due to the fact that it would consume a huge amount of time and make any changes to the training samples prohibitive.

OTB offers numerous classifiers, each one having discrete features. For both cases (raster and vector classification), a supervised classification was implemented and SVM algorithm was employed for training. SVM algorithm has revealed its effectiveness in numerous remote sensing projects, showing excellent performance (De Luca et al., 2019).

The goal of classification is to determine in each of the segmented image of the study area the land cover class for all the image objects. In order to train the classifier, the selection of land cover classes was carried out a priori and are the following:

1. Vegetation sparse

2. Vegetation dense

3. Built-up area

4. Roads

5. Cultivated land

6. Barren land

7. Shadows (only in QuickBird image)
Samples of confirmed land cover need to be selected that will train the classifier. They ought to be chosen from across the scene and not a specific area (Wang and Campbell, 2018). The selection of training areas for each land cover class was based on personal knowledge of the study area and visual interpretation with the use of Google Earth historical dataset in order to be of the similar time period as the satellite data. The final classification outputs based on the aforementioned land cover classes, are presented in Figures 9-10-11.

Accuracy assessment of the obtained classification results was conducted in Orfeo ToolBox, in order to investigate the validity of results. The accuracy was evaluated using overall accuracy, producer's accuracy and user's accuracy values through an error matrix which compares the information from the classified image with reference data. The error matrices are presented in Table 2.

\section{DISCUSSION AND CONCLUSIONS}

For very high resolution imageries, it seems more logical and effective to classify segments rather than pixels. At the same time, fewer and fewer Remote Sensing scientists support that further improvements in satellite sensors' spatial resolution will provide more accurate results. The objective of this paper is to present the basic principles regarding OBIA and a methodological framework in order to classify land cover not only in a very high but also in a moderate and high spatial resolution image. OBIA may be in some manner the linkage between the Remote Sensing science and GIS science. The most important asset of object-based image classification is that it can be conducted very fast and the outcomes are sufficiently accurate. There is not a specific algorithm that will produce the best results for all images, so the parameters values selected are indicative for a study area that covers a combination of urban and suburban landscapes. Continuous monitoring of this area through obtaining land cover thematic maps and comparing them in time presents a great interest, but this composes the subject of a future study.

The analysis showed that Sentinel-2A data performs better than Landsat-8 and QuickBird data, when performing land cover classification in an urban and suburban environment. Shadows seem to be a major noise for QuickBird data, while Landsat low accuracy is attributed to the major limitation of OBIA on Landsat land cover classification which is the low spatial resolution of Landsat images. The extraction of land cover types with relatively small area such as road network demands higher levels of spatial analysis, which Landsat data do not offer. On the other hand, the results may be acceptable when performing regional scale mapping. To sum up, OBIA works well with Landsat- 8 and QuickBird data with overall accuracies above $80 \%$ and in the case of Sentinel-2A it works exceptionally with over $90 \%$ overall accuracy.

Nevertheless, the satellite data and method selected for classifying land cover should be an objective decision depending on what is required and what kind of results are anticipated. For future research, a common technique for any type of study area can be found, but such a task is very challenging since every landscape has its unique characteristics. 

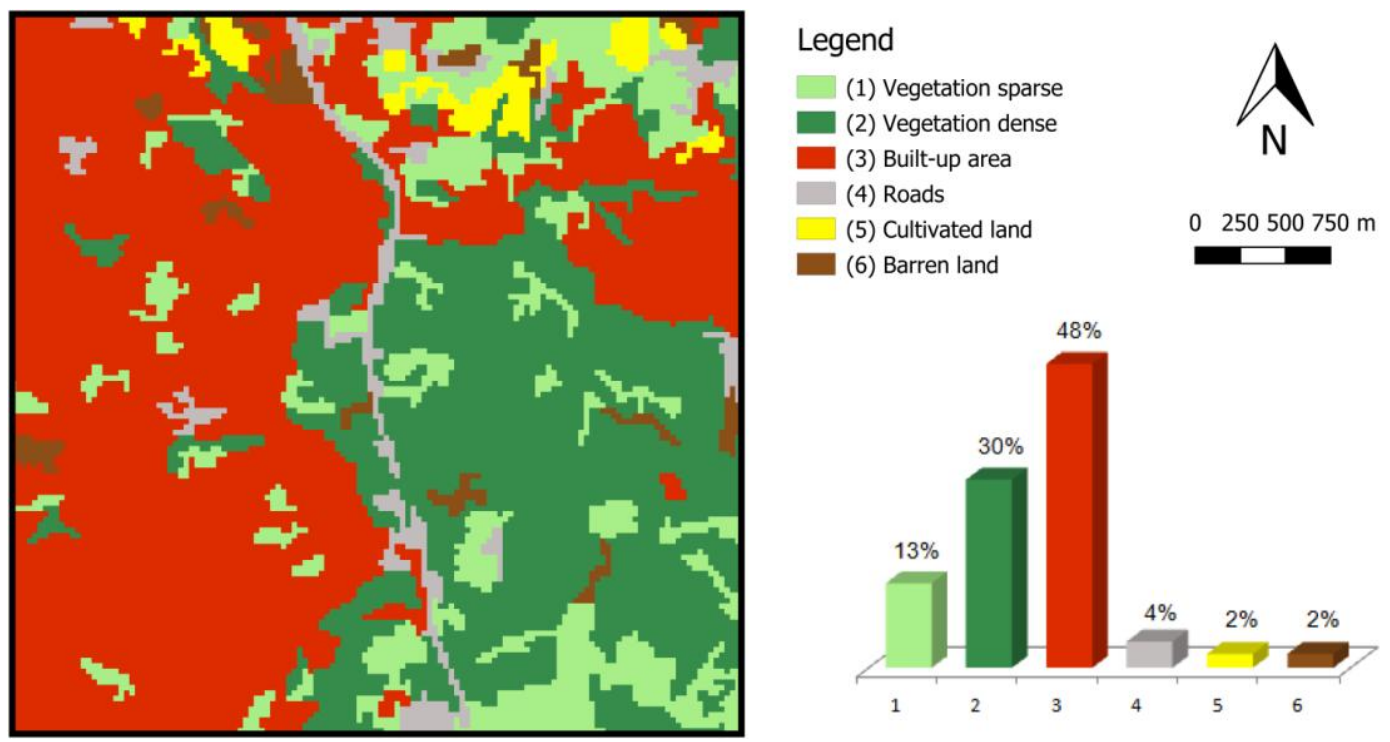

Figure 9. Landsat-8 classified image
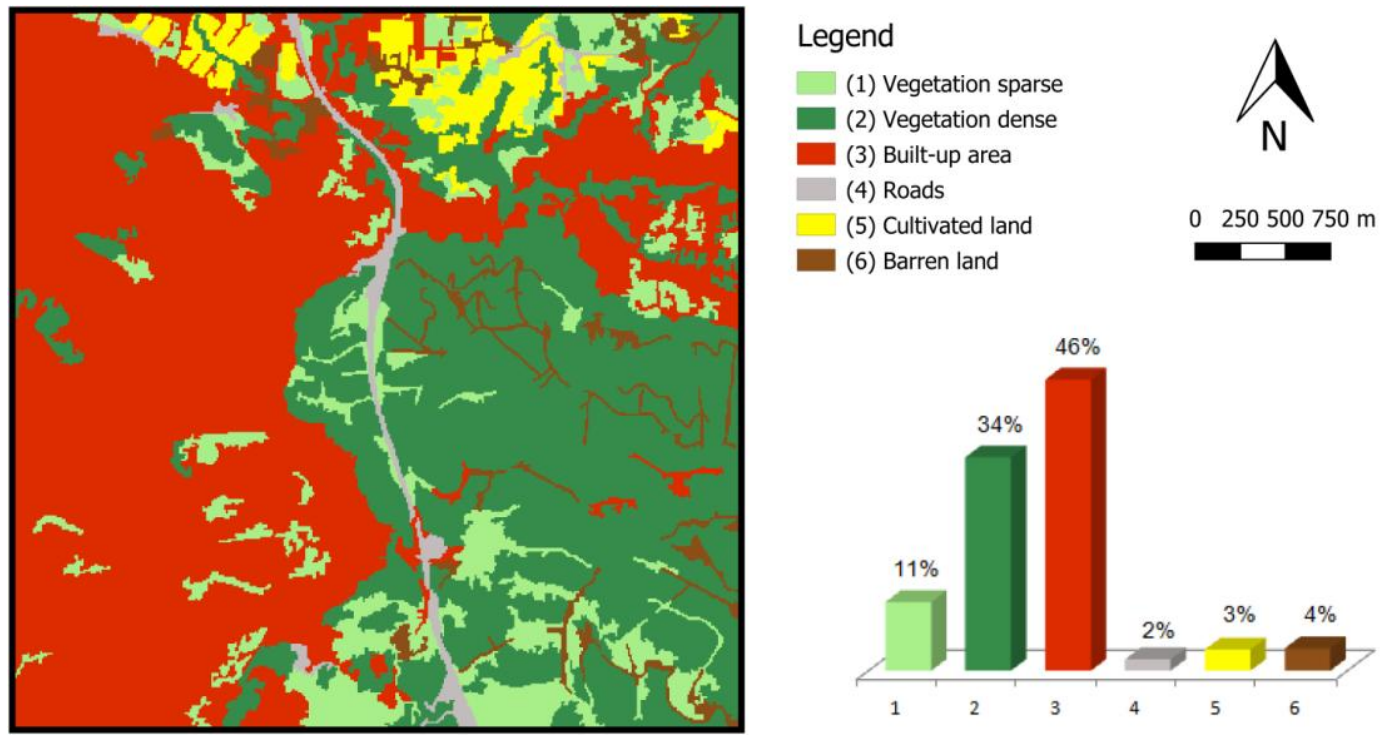

Figure 10. Sentinel-2A classified image
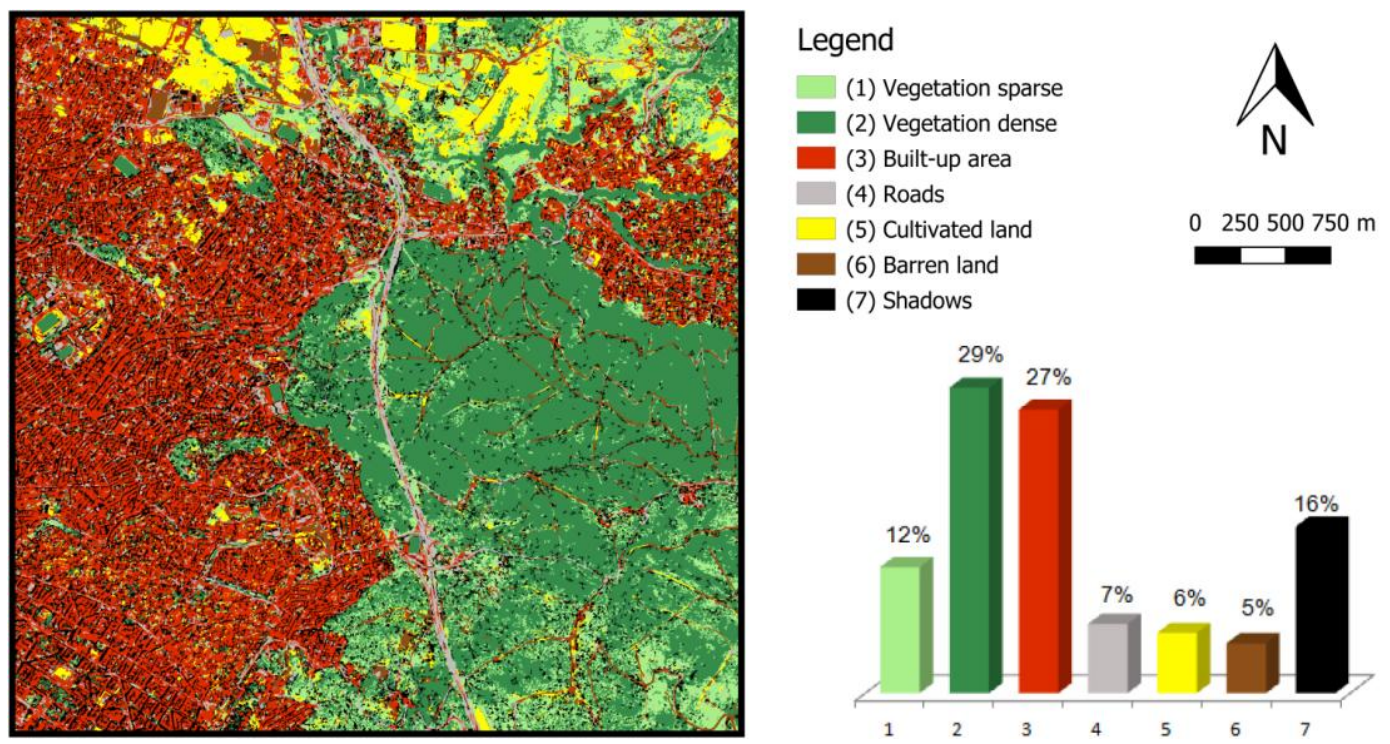

Figure 11. QuickBird classified image 


\section{Reference labels}

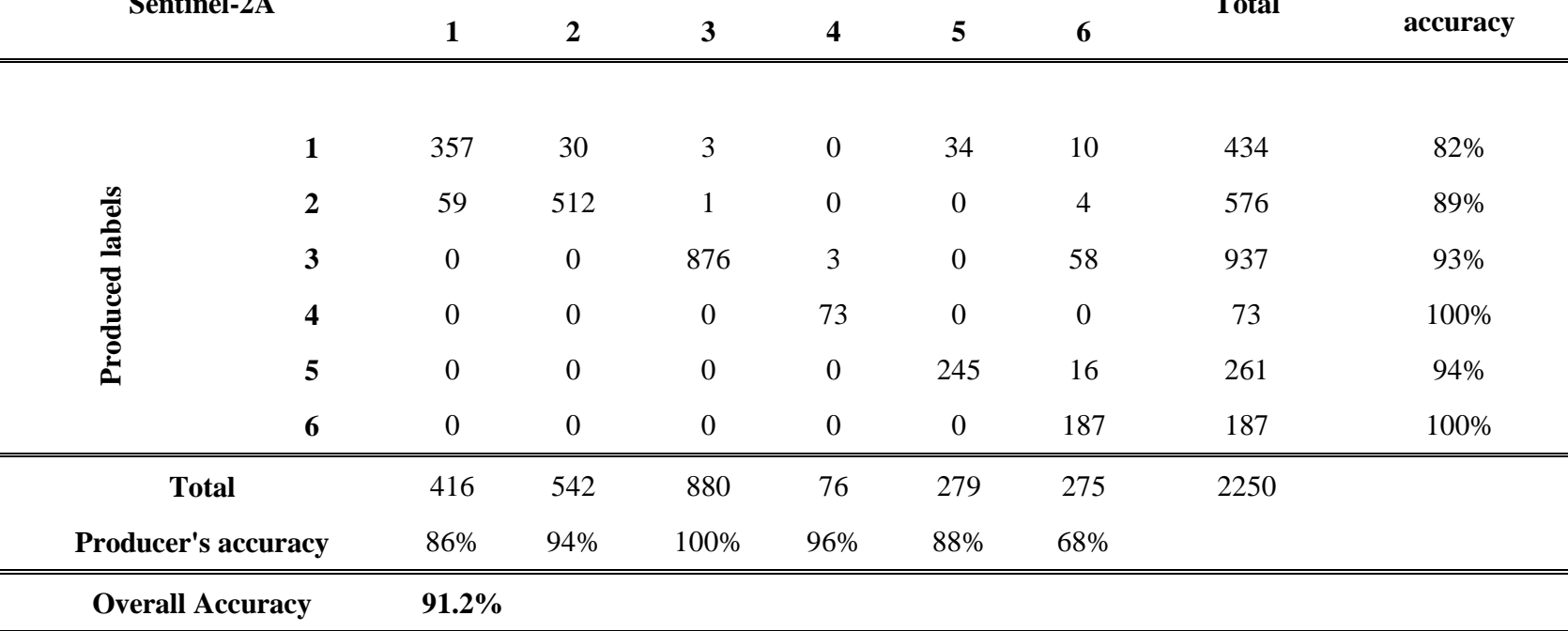

Landsat-8

Reference labels

\begin{tabular}{|c|c|c|c|c|c|c|c|c|c|}
\hline \multirow{5}{*}{ 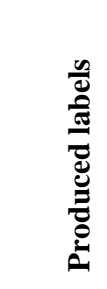 } & 1 & 44 & 0 & 11 & 0 & 2 & 9 & 66 & $67 \%$ \\
\hline & 2 & 0 & 63 & 0 & 0 & 1 & 0 & 64 & $98 \%$ \\
\hline & 3 & 0 & 3 & 104 & 0 & 2 & 3 & 112 & $93 \%$ \\
\hline & 4 & 2 & 7 & 0 & 9 & 0 & 0 & 18 & $50 \%$ \\
\hline & 5 & 0 & 0 & 0 & 0 & 25 & 2 & 27 & $93 \%$ \\
\hline & 6 & 0 & 0 & 22 & 0 & 4 & 30 & 56 & $54 \%$ \\
\hline \multicolumn{2}{|c|}{ Total } & 46 & 73 & 137 & 9 & 34 & 44 & 275 & \\
\hline \multicolumn{2}{|c|}{ Producer's accuracy } & $96 \%$ & $86 \%$ & $76 \%$ & $100 \%$ & $74 \%$ & $68 \%$ & & \\
\hline
\end{tabular}

Overall Accuracy $\quad \mathbf{8 0 . 2 \%}$

QuickBird
Reference labels

1
1

$\begin{array}{lll}2 & 3 & 4\end{array}$

$\begin{array}{lll}4 & 5 & 6\end{array}$

Total

User's accuracy

\begin{tabular}{|c|c|c|c|c|c|c|c|c|c|c|}
\hline \multirow{2}{*}{\multicolumn{2}{|c|}{ QuickBird }} & \multicolumn{6}{|c|}{ Reference labels } & \multirow[b]{2}{*}{7} & \multirow{2}{*}{ Total } & \multirow{2}{*}{$\begin{array}{c}\text { User's } \\
\text { accuracy }\end{array}$} \\
\hline & & 1 & 2 & 3 & 4 & 5 & 6 & & & \\
\hline \multirow{7}{*}{ 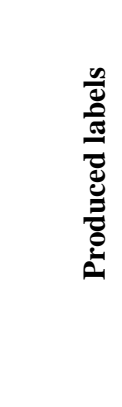 } & 1 & 25654 & 4654 & 112 & 49 & 5487 & 3354 & 537 & 39847 & $64 \%$ \\
\hline & 2 & 486 & 101579 & 95 & 33 & 0 & 0 & 0 & 102193 & $99 \%$ \\
\hline & 3 & 0 & 232 & 57000 & 2624 & 252 & 2897 & 135 & 63140 & $90 \%$ \\
\hline & 4 & 487 & 312 & 6029 & 11706 & 367 & 582 & 2 & 19485 & $60 \%$ \\
\hline & 5 & 219 & 0 & 10264 & 390 & 41385 & 4781 & 0 & 57039 & $73 \%$ \\
\hline & 6 & 0 & 452 & 119 & 0 & 2763 & 33878 & 0 & 37212 & $91 \%$ \\
\hline & 7 & 146 & 1894 & 3390 & 294 & 0 & 121 & 9085 & 14930 & $61 \%$ \\
\hline \multicolumn{2}{|c|}{ Total } & 26992 & 109123 & 77009 & 15096 & 50254 & 45613 & 9759 & 280287 & \\
\hline \multicolumn{2}{|c|}{ Producer's accuracy } & $95 \%$ & $93 \%$ & $74 \%$ & $78 \%$ & $82 \%$ & $74 \%$ & $93 \%$ & & \\
\hline
\end{tabular}

Overall Accuracy $\quad \mathbf{8 4 . 0 \%}$

Table 2. Confusion matrices with land cover types indicated as follows: $1=$ Vegetation sparse, $2=$ Vegetation dense, $3=$ Built-up area, 4=Roads, 5=Cultivated land, $6=$ Barren land, 7=Shadows 


\section{REFERENCES}

Blaschke, T., Lang, S., Hay, G. (Eds.), 2008. Object-Based Image Analysis: Spatial Concepts for Knowledge-Driven Remote Sensing Applications, Lecture Notes in Geoinformation and Cartography. Springer-Verlag Berlin Heidelberg, Berlin, Heidelberg. doi.org/10.1007/978-3-540-77058-9.

Blaschke, T., 2010. Object based image analysis for remote sensing. ISPRS Journal of Photogrammetry and Remote Sensing 65, 2-16. doi.org/10.1016/j.isprsjprs.2009.06.004.

Chehata, N., Orny, C., Boukir, S., Guyon, D., Wigneron, J.P., 2014. Object-based change detection in wind storm-damaged forest using high-resolution multispectral images. International Journal of Remote Sensing 35(13), 4758-4777. doi.org/10.1080/01431161.2014.930199.

Christophe, E., Inglada, J., Giros, A., 2008. Orfeo toolbox: a complete solution for mapping from high resolution satellite images. The International Archives of the Photogrammetry, Remote Sensing and Spatial Information Sciences XXXVII (Part B4), 1263-1268.

Csillik, O., Belgiu, M., 2017. Cropland mapping from Sentinel2 time series data using object-based image analysis, in: Societal Geo-Innovation: Short Papers, Posters and Poster Abstracts of the 20th AGILE Conference on Geographic Information Science. Wageningen, the Netherlands.

De Luca, G., Silva, J.M.N., Cerasoli, S., Araújo, J., Campos, J., Di Fazio, S., Modica, G., 2019. Object-Based Land Cover Classification of Cork Oak Woodlands using UAV Imagery and Orfeo ToolBox. Remote Sensing 11, 1238. doi.org/10.3390/rs11101238.

DigitalGlobe, Inc., 2003. QuickBird Imagery Products - Product Guide. genesiis.com/alpha/pdf/quickbird-product-guide.pdf (25 April 2020).

Grizonnet, M., Michel, J., Poughon, V., Inglada, J., Savinaud, M., Cresson, R., 2017. Orfeo ToolBox: open source processing of remote sensing images. Open geospatial data, Software and Standards 2 (1), 15. doi.org/10.1186/s40965-017-0031-6.

Jensen, J. R., \& Cowen, D. C., 1999. Remote Sensing of Urban/Suburban Infrastructure and Socio-Economic Attributes. Photogrammetric engineering and remote sensing 65(5), 611-622.

Kaplan, G., Avdan, U., 2017. Object-based water body extraction model using Sentinel-2 satellite imagery. European Journal of Remote Sensing 50(1), 137-143. doi.org/10.1080/22797254.2017.1297540.

Labib, S.M., Harris, A., 2018. The potentials of Sentinel-2 and LandSat-8 data in green infrastructure extraction, using object based image analysis (OBIA) method. European Journal of Remote Sensing 51(1), 231-240. doi.org/10.1080/22797254.2017.1419441.

Michel, J., Grizonnet, M., Jaen, A., Harasse, S., Hermitte, L., Guinet, J., Malik, J. \& Savinaud, M., 2012. Open tools and methods for large scale segmentation of very high resolution satellite images. OGRS2012, 179-184.
Novelli, A., Aguilar, M.A., Nemmaoui, A., Aguilar, F.J., Tarantino, E., 2016. Performance evaluation of object based greenhouse detection from Sentinel-2 MSI and Landsat 8 OLI data: A case study from Almería (Spain). International Journal of Applied Earth Observation and Geoinformation 52, 403411. doi.org/10.1016/j.jag.2016.07.011.

Nussbaum, S., Menz, G., 2008. Object-based image analysis and treaty verification: new approaches in remote sensing applied to nuclear facilities in Iran. Springer, New York, NY. doi.org/10.1007/978-1-4020-6961-1.

Orfeo ToolBox, 2020. OTB CookBook. orfeotoolbox.org/packages/doc/test-cookbookworkshops/index_TOC.html (25 April 2020).

Phiri, D., Morgenroth, J., 2017. Developments in Landsat Land Cover Classification Methods: A Review. Remote Sensing 9 (9), 967. doi.org/10.3390/rs9090967.

Pozzi, F., Small, C., 2001. Exploratory analysis of suburban land cover and population density in the U.S.A. IEEE/ISPRS Joint Workshop on Remote Sensing and Data Fusion over Urban Areas, $\quad$ Rome, Italy. doi.org/10.1109/DFUA.2001.985890.

Remote Sensing for Forest Cover Change Detection, 2016. Module 3: Introduction to QGIS and Land Cover Classification. servirglobal.net/Portals/0/Documents/Articles/ChangeDetection Training/Module3_LC_Classification_Accuracy_Assessment.p df (25 April 2020).

Thenkabail, P.S. (Ed.), 2015. Remotely Sensed Data Characterization, Classification, and Accuracies. CRC Press, Boca Raton, Florida. doi.org/10.1201/b19294.

Topouzelis, K., Makri, D., Stoupas, N., Papakonstantinou, A., Katsanevakis, S., 2018. Seagrass mapping in Greek territorial waters using Landsat-8 satellite images. International Journal of Applied Earth Observation and Geoinformation 67, 98-113. doi.org/10.1016/j.jag.2017.12.013.

Wang, Y.Q., Campbell, A., 2018. Object-based Image Analysis (OBIA) of Salt Marshes: Standard Operating Procedures (Version 1.0). Northeast Coastal and Barrier Network, National Park Service, Kingston, RI irma.nps.gov/Datastore/DownloadFile/604252 (25 April 2020). 